\title{
How can Improve DNA Vaccine Modalities as a Therapeutic Approach against HIV Infections?
}

Nourieh Habibzadeh ${ }^{1,2}$, Azam Bolhassani ${ }^{1 *}$, Rouhollah Vahabpour ${ }^{1}$ and Seyed Mehdi Sadat ${ }^{1}$

${ }^{1}$ Department of Hepatitis and AIDs, Pasteur Institute of Iran, Tehran, Iran

'Islamic Azad University of Pharmaceutical Sciences Branch, Tehran, Iran

\begin{abstract}
The recent studies have focused on the improvement of effective prophylactic and therapeutic approaches to combat persistent viral infections. Several therapeutic vaccine strategies have already used such as live-attenuated microbes, viral vectors and dendritic cell-based vaccines led to suppress and/or clear infections. Among them, improved DNA vaccines have emerged as a promising candidate for treatment of infectious diseases especially HIV infections. These vaccines have the advantages of safety, low cost of production, ease of use due to their stability in room temperature as well as ability to elicit efficient immune responses. Some strategies have been considered to improve immune responses stimulated by DNA vaccines, e.g., genetic optimization of plasmid DNA constructs encoding antigens, in vivo efficient DNA delivery systems, co-delivery with molecular adjuvants as well as the development of potent heterologous prime-boost regimens. DNA vaccines have an intrinsic bias towards generating cellular immunity against intracellular pathogens. By manipulating the DNA formulation and delivery, effective antibody responses can be also induced. For instance, the studies showed that the immunized monkeys with DNA vaccine developed HIVspecific T-cell immune responses that persisted for months. It is important to develop the potency of this modality in the clinic. In this review, the authors will focus on the recent improvements to enhance DNA vaccine potency in combating persistent HIV virus infections.
\end{abstract}

Keywords: DNA vaccine; Infectious disease; HIV; Adjuvant; Delivery system; Prime-boost regimen

\section{Introduction}

Nucleic acid immunization (DNA/ RNA) is a novel method for development of vaccines against various infectious diseases [1,2]. The first DNA vaccines for prevention of infectious diseases were explained in 1993 and have been indicated to generate protective humoral and cellular immune responses to different infectious agents [3]. Compared with live viral or bacterial vectors, plasmid DNA is relatively safe, can be easily administered, is easy to prepare on a large scale with high purity and stability, and can be engineered to express antigenic peptides or proteins. However, a major problem of the DNA vaccine is its limited potency to induce an immune response [4,5]. Thus, optimal strategies to enhance the efficiency of DNA immunization remain to be determined [5]. Different vaccines tend to induce the specific type of immune responses. Protein vaccines tend to stimulate Th2 type responses while DNA vaccines produce further Th1 type responses [2,68]. For example, production of Th1-type cytokines (IL-2, IFN- $\gamma$ ) and IgG2a isotype (a Th1 indicator) was increased by HSV DNA primingprotein boosting similar to DNA-DNA vaccination. In contrast, protein priming-DNA boosting generated antibody levels (especially, an increased IgG1 isotype as a Th2 indicator) similar to protein-protein vaccination but failed to produce cytokines. This result indicates that preferential induction of immune responses (e.g., IgG1 or IgG2a isotype) is determined by the first administration (i.e., type of priming) [9]. Optimization of the immune responses can be achieved through various approaches to increase DNA vaccine potency, such as vector modification, adjuvants and facilitation of DNA delivery [2,6-8]. Coinjection of adjuvants is one approach to increase the amplitude of the immune response, and to bias it toward Th1 or Th2 using DNA vaccines in humans [10]. This approach enables localized expression of potent molecules at the site of antigen expression and stimulation of immune responses (e.g., genetic adjuvants such as cytokines, chemokines and $\mathrm{T}$ cell co-stimulatory molecules as a component of the vaccine) $[10,11]$. On the other hand, delivery of plasmid DNA vaccines using physical delivery systems (e.g., particle mediated epidermal delivery, gene gun, electroporation) reduces the amount of plasmid DNA needed to induce immune responses. In this line, Langerhans cells are directly transfected following these routes of delivery. The direct transfection of Langerhans cells is thought to elicit immune responses with very small doses of plasmid DNA ( 1-10 $\mu \mathrm{g})$. For example, the immunogenicity of intramuscular plasmid DNA injection could be developed by electroporation [12]. Heterologous prime-boost regimens are also especially potent at inducing cellular immune responses and have been used to solve the issue of pre-existing vector immunity. The advantage of heterologous prime-boost immunization regimens to induce robust antigen-specific $\mathrm{CD} 8^{+} \mathrm{T}$ cells has been confirmed in many preclinical models. In humans, heterologous prime-boost immunization regimens are currently being tested for cancer (melanoma, colon carcinoma, prostate carcinoma) and infectious diseases [12].

Human immunodeficiency virus (HIV) causes acquired immunodeficiency syndrome (AIDS) and remains one of the most serious threats to global health [13]. Despite efforts over the past several years, a successful HIV vaccine still remains obscure. The studies showed that some problems like pre-existing immunity, as observed with viral vectors such as Adenovirus type 5 (Ad5), Adeno Associated Virus (AAV) and Modified Vaccinia Ankara (MVA) can be

*Corresponding author: Azam Bolhassani, Assistant Professor, Department of Hepatitis and AIDs, Pasteur Institute of Iran, Tel: +98 21 66953311; Fax: +98 21 66465132; E-mail: azam.bolhassani@yahoo.com

Received January 24, 2015; Accepted March 06, 2015; Published March 15 2015

Citation: NHabibzadeh, Bolhassani A, Vahabpour R, Sadat SM (2015) How can Improve DNA Vaccine Modalities as a Therapeutic Approach against HIV Infections? J AIDS Clin Res 6: 440. doi:10.4172/2155-6113.1000440

Copyright: (c) 2015 Habibzadeh N, et al. This is an open-access article distributed under the terms of the Creative Commons Attribution License, which permits unrestricted use, distribution, and reproduction in any medium, provided the original author and source are credited. 


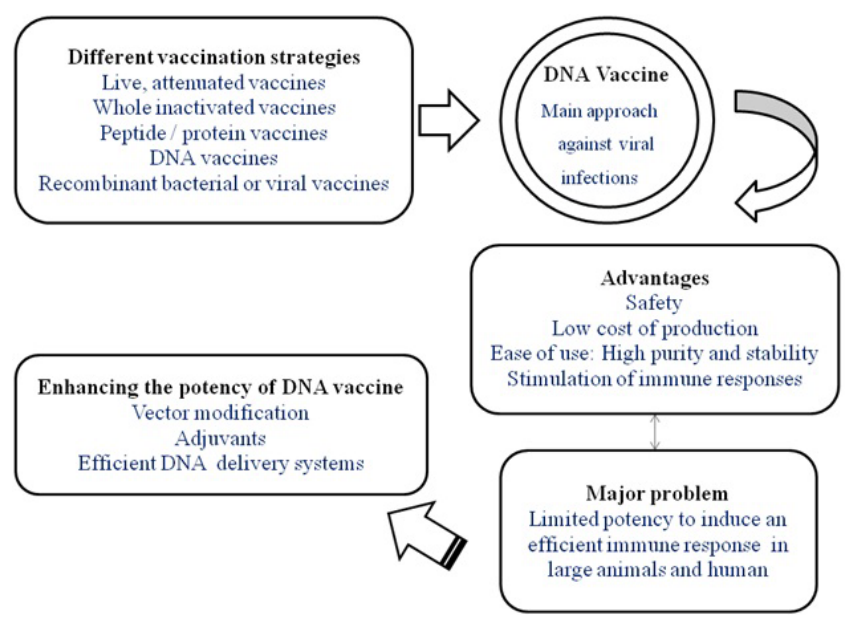

Figure 1: Overview of DNA vaccine strategy.

avoided with DNA-based vaccines. Nevertheless, optimization of both the immunogens and vaccination strategies will be needed to generate potent antibodies especially neutralizing antibodies (NAb). Due to the diversity of HIV, an effective viral envelop (Env)-based vaccine will likely need a broad coverage of antigenic variants. A vaccine codelivering Env immunogens as DNA and protein components could provide such coverage [14]. This review is focused on optimization of DNA-based vaccines using different strategies against HIV infections. Figure 1 shows an overview of DNA vaccination.

\section{Structure and Function of DNA Vaccine}

DNA vaccines are composed of bacterial plasmids that have been engineered to express the disease-specific antigen using promoter elements that are active in mammalian cells. They also include a transcriptional terminator to terminate transcription in mammalian cells and a selectable marker to facilitate production of the plasmids in transformed bacterial cells [11]. The antigen encoded by the plasmid DNA is produced either in professional antigen presenting cells (APCs) leading to direct priming of immune responses or in nonprofessional cells from where the antigen can be transferred to APCs leading to cross-priming. In addition, DNA plasmids stimulate the innate immune system by interacting with Toll-like receptor 9 (TLR 9). This non-specific immune response enhances the antigen-specific immune responses [12]. The DNA fusion vaccine strategy has proved to be efficient against a variety of antigens using various molecules with different immunological properties or functions. Indeed, DNA fusion vaccines incorporating various immune-enhancing molecules can be used to elicit immunity against attached antigens [15]. Regarding to the potent effects of DNA fusion vaccines, our research group has designed a fusion plasmid DNA expressing MPER-V3 protein for improving immunological effects in mice model (unpublished data). The HIV-1 gp41 MPER contains the epitopes for broadly neutralizing antibodies (NAbs), making it as a target for HIV vaccine design. The studies indicated that DNA vaccines expressing the MPER of HIV-1 gp41 induce different antibodies depending on their transmembrane and cytoplasmic domains [16]. In addition, neutralizing antibodies, which recognize the main neutralizing determinants of the gp120 Env protein (e.g., the V3 loop region), have been shown to effectively block cell fusion and virus infectivity independent of the initial gp120-CD4 binding [17]. Thus, this region can broadly develop NAbs in HIV DNA vaccine design.

\section{DNA Vaccine Delivery}

A successful prophylactic HIV-1 vaccine would elicit persistent humoral and cellular immune responses in immunized individuals. From the obtained studies, it is clear that DNA immunization by intramuscular (IM) or intradermal (ID) route elicits poor antibody and T-cell responses that can be boosted by other vaccine regimens including subunit proteins. In addition, plasmids delivered by IM or ID routes can remain at the site of injection for prolonged periods. Therefore, lower doses of DNA would potentially facilitate clearance of plasmids from injection site quickly resulting in lower risks of toxicity [18]. To enhance immunological priming by DNA vaccine, physical delivery by electroporation (EP) has further been studied $[18,19]$. Electroporation utilizes a small electric field across the site of injection (the muscle or skin) causing temporary membrane instability and also an electric gradient, which increases the cellular uptake of DNA $[13,19,20]$. EP could overcome the failure of low-volume inoculation to induce immunity in mice, and the combination of DNA priming with DNA/EP at boosting was remarkably effective. Also, EP significantly indicated an improved vaccine-specific $\mathrm{T}$-cell responses and a reduction of viremia in rhesus macaques [20]. In macaques, electroporation of low dose DNA encoding HIV-1 env followed by gp120 protein elicited Th1 cytokines and functional CTL that continued for over one year. These data indicated efficient priming of immune responses by electroporated DNA that in conjunction with protein boost may lead to long-term immunity in immunized hosts [18]. Moreover, the electroporation technology was similarly effective in rhesus macaques for enhancing immune responses against HIV Gag and Env after DNA vaccination. A comparative study showed that although, DNA delivery by gene gun is highly effective in eliciting immune responses with low dose of immunogen, such responses are often skewed towards Th2 responses. In contrast, Th1 responses and CTL activity were elicited by low doses of electroporated DNA [18]. On the other hand, broad CTL response against HIV-1 is one factor that helps to control the viral replication [21]. In other study, a single gag-pol fusion DNA vaccine by intramuscular electroporation was sufficient for eliciting immune responses against both gag and pol antigens [22]. As known, ADVAX is a multigenic DNA-based candidate HIV vaccine that was safe but weakly immunogenic when delivered intramuscularly in humans. Studies were performed in animal models to determine whether in vivo electroporation (EP), could improve the immunogenicity of ADVAX while maintaining an acceptable safety profile. Mice immunization by ADVAX with or without EP revealed significantly higher IFN- $\gamma$ responses to all antigens in the EP groups. Furthermore, PCR based quantitation of residual plasmid indicated that the potential for integration events into the host genome was low. These data suggested the clinical development of ADVAX delivered by EP in humans [23].

\section{Live Attenuated Vaccine}

The use of live attenuated invasive bacteria as a carrier for DNAbased vaccines has previously been reported [24]. Immunization with recombinant invasive bacteria including Shigella, Salmonella and Listeria carrying plasmid DNA vaccines has been shown to induce protective immune responses in mice. The use of human enteric bacteria is especially useful due to their ability to infect human colonic mucosa, and their tropism for activation of dendritic cells and macrophage of internal mucosa. Thus, they are very efficient for delivery of DNA vaccines to APCs in the mucosa resulting in stimulation of potent systemic and local immune responses. Such responses may be critical for the development of an effective prophylactic HIV vaccine, because a large number of HIV transfer through human mucosal routes [24]. 
For instance, a live attenuated strain of Salmonella typhimurium was used to deliver plasmid orally and showed an adjuvant role through the release of various cytokines [25]. In addition, intranasal immunization of mice with live recombinant Shigella cells induced a HIV gag-specific cellular immune response similar to that observed by IM injection of naked DNA. Importantly, a strong boosting effect was obtained in mice primed with DNA, suggesting the efficacy of bacterial vectors in primeboost vaccination regimens [24]. The studies indicated that a novel vaccine delivery system using bacterial ghosts (BGs) can be considered as an efficient and non-toxic delivery system for DNA vaccines in vitro and in vivo. In this line, a new strategy of HIV vaccine delivery was designed using Salmonella typhi Ty21a bacterial ghosts (BGs). The data showed that Ty21a BGs loaded with an HIV gp140 DNA vaccine (Ty21a BG-DNA) are easily taken up by murine macrophage cells (RAW264.7), and gp140 is efficiently expressed in these cells. Peripheral and intestinal mucosal anti-gp120 antibody responses in mice vaccinated with BGs-DNA vaccine were significantly higher than those in mice immunized with naked DNA vaccine. The enhancement of antibody responses was associated with BG-induced production of IL-10 through TLR4 pathway [26].

Attenuated virus vaccines have traditionally been potent and relatively easy to produce and deliver. Vaccination with a live virus results in high intracellular synthesis of viral proteins. This high-level expression stimulates strong cellular and humoral immune responses and results in the production of long-lasting memory $\mathrm{B}$ and $\mathrm{T}$ cells. However, attenuated HIV vaccines replicate strongly in animal models to retain residual virulence. Recent studies indicated that priming with a DNA vaccine induces a Th1 response that can be boosted by the subsequent administration of a viral vector encoding the same gene. This prime-boost strategy elicited strong protective immunity in several primate models [27]. Currently, immunogenicity of a highly attenuated vaccinia virus with low neuro-virulence, $\mathrm{LC} 16 \mathrm{~m} 8$ strain was studied as an HIV vaccine vector. The data showed that the recombinant vaccinia virus-based vaccine (vLC-Env) combined with DNA vaccine expressing the HIV env gene (pCAG-Env) produces a protective immune response against HIV infection in BALB/c mice. Vaccination of vLCEnv alone induced much higher HIV-specific humoral and cellular immune responses than that of pCAG-Env. Priming with pCAG-Env further enhanced vLC-Env induced immune responses, especially cell-mediated immune response. In addition, administration of vLCEnv-infected dendritic cells to mice generated a high cellular immune response. These results demonstrated that priming with pCAG-Env and boosting with vLC-Env represents a potential candidate for vaccination against HIV infection [27].

\section{Adjuvants}

Although the importance of DNA vaccines, especially as a priming immunization has been well proved in different HIV vaccine studies, the immunogenictiy of DNA vaccines is generally moderate. Novel adjuvant is necessary for improving the immunogenicity of DNA vaccine [28]. Multiple groups have demonstrated the potential of co-administering plasmid DNA (pDNA) that express cytokines, chemokines or co-stimulatory molecules together with plasmids encoding target viral antigens [29]. The potency of DNA vaccines can be developed by the co-delivery of plasmid-encoded molecular adjuvants. The pDNAs encoding granulocyte-macrophage colony stimulating factor (GM-CSF), hematopoietic factor fms-like tyrosine kinase 3 ligand (Flt-3L), interleukin-12 (IL-12) could markedly enhance the cell-mediated immune responses elicited by an HIV-1 env pDNA vaccine in BALB/c mice [29]. Plasmid GM-CSF also increased the immune responses elicited by DNA vaccines expressing HIV-1 Gag and Nef-Tat-Vif. In addition, the use of pGM-CSF as a vaccine adjuvant appeared to increase antigen-specific proliferative responses and the percentage of polyfunctional memory $\mathrm{CD}^{+} \mathrm{T}$ cells. Co-delivery of pFlt-3L with pGM-CSF did not result in a further increase in adjuvant activity. However, the co-administration of pGM-CSF with pIL-12 significantly enhanced env-specific proliferative responses and vaccine efficacy in the murine vaccinia virus challenge model relative to mice immunized with the env pDNA vaccine adjuvanted with either pGMCSF or pIL-12 alone [29]. In another study, co-administration of the HIV-1 DNA vaccine with pIL-12 and pGM-CSF by topical application to the skin enhanced the levels of both the HIV-specific cytotoxic T lymphocyte response and delayed-type hypersensitivity (DTH). Indeed, the skin is accessible for generating immune responses by both intradermal injection and topical use of gene delivery vectors [30]. In addition, co-administration of plasmids encoding the codon-optimized GM-CSF sequence with the HIV-1 Gag DNA vaccine resulted in a strong antibody and CTL response and a protective immune response against infection with recombinant vaccinia virus expressing HIV-1 Gag [31]. On the other hand, the researchers strongly support the use of IL-6 or IL-15 as a cytokine adjuvant in HIV DNA vaccination. The data indicated that intranasal administration of DNA vaccine and pIL15 is able to enhance the Th1 dependent HIV-1-specific cell-mediated immunity. However, co-injection of pIL-15 with pIL-2 or pIL-12 did not show any synergistic effect on the immune responses induced by DNA vaccine in vivo [32]. Furthermore, the immunogenicity of HIV1 DNA vaccine expressing the chimeric gene gag-gp120 (pVAX1-gaggp120) was increased by co-inoculating pVAX1-IL6 in BALB/c mice [33]. The studies demonstrated that in-frame fusion of tumor necrosis factor alpha (TNF- $\alpha$ ) DNA to DNA encoding a large fragment of HIV gp120 could enhance Th1 immune responses against gp120 antigen. Also, in-frame fusion of IFN $\gamma$-encoding DNA at the 5 ' end of the chimeric molecule, to create a tripartite fusion, had no additional effect on immunogenicity [34].

A number of studies have shown that a-galactosylceramide ( $a$-GalCer), a natural killer T cell (NKT) ligand, was applied as an adjuvant for various vaccines, including viral, parasite and proteinbased vaccines. The $\alpha$-GalCer was able to enhance HIV-specific antibody responses. Furthermore, co-administration of $\alpha$-GalCer with suboptimal doses of DNA vaccines greatly increased antigen-specific $\mathrm{CD}^{+}$and $\mathrm{CD}^{+} \mathrm{T}$ cell responses. The level of cell-mediated immune responses in mice vaccinated with $5 \mu \mathrm{g}$ of DNA in the presence of $\alpha$-GalCer was similar to that of mice vaccinated with $50 \mu \mathrm{g}$ of DNA in the absence of $\alpha$-GalCer [35].

Recombinant adjuvants composed of a fusion between Surfactant Protein D (SP-D) and either CD40 Ligand (CD40L) or GITR Ligand (GITRL) were previously shown to enhance HIV-1 Gag DNA vaccines. It was demonstrated that similar fusion constructs composed of the TNF superfamily ligands (TNFSFL) including 4-1BBL, OX40L, RANKL, LIGHT, CD70, and BAFF can also enhanced immune responses to a HIV-1 Gag DNA vaccine. Importantly, the SP-D-4-1BBL, SP-D-OX40L, and SP-D-LIGHT constructs enhanced $\mathrm{CD}^{+} \mathrm{T}$ cell avidity and $\mathrm{CD}^{+} /$ $\mathrm{CD} 4^{+} \mathrm{T}$ cell proliferation seven weeks after vaccination [36]. Also, the SP-D-OX40L, SP-D-LIGHT, and SP-D-BAFF constructs increased Gag-specific IL-2 secretion in memory T cells, suggesting their potency to elevate the number of self-renewing Gag-specific $\mathrm{CD} 8^{+}$and $\mathrm{CD} 4^{+} \mathrm{T}$ cells. Finally, the SP-D-OX40L and SP-D-CD70 adjuvants augmented IgG2a but not IgG1 antibody responses in the immunized animals. Interestingly, the B cell-activating protein BAFF did not enhance antiGag antibody responses when administered as an SP-D fusion adjuvant, 
but augmented $\mathrm{CD}^{+}$and $\mathrm{CD} 8^{+} \mathrm{T}$ cell responses. Indeed, various SP-DTNFSFL fusion constructs can enhance immune responses following DNA vaccination with HIV-1 Gag expression plasmid [36].

Several studies indicated that $4-1 \mathrm{BB}$ and $4-1 \mathrm{BB}$ ligand (4-1BBL) interactions are important for inducing robust CTL responses and also long-lived memory T-cells. Recently, the plasmid DNAs expressing either the membrane bound or soluble form of 4-1BBL were designed to enhance the Gag DNA vaccine as an adjuvant. The data showed that 4-1BBL DNA increased the Gag-specific IgG and cellular immune responses. Importantly, the expression of Gag and 4-1BBL from the same plasmid was critical for the adjuvant activity [11].

To improve the immunogenicity of DNA vaccines, some studies were focused on the immunoglobulin (Ig) fusion antigen. These reports showed that cytokine-coding plasmids fused with Ig have higher expression efficiency and better adjuvanticity. Furthermore, these plasmids have features that make them useful such as augmentation of half life in vivo, formation of a multivalent antigen, and solubilization of hydrophobic proteins [37]. The possibility of increasing HIV gp120specific cellular immune responses was determined in mice using a DNA vaccine encoding a mouse Ig fragment fused with gp120 in two directions (gp120-Ig or Ig-gp120). In vitro expression analysis revealed that the efficiency of HIV gp120 protein expression was higher in cells transfected with the gp120-Ig-coding plasmid (pGp120Ig) than in those transfected with the gp120 and Ig-gp120 expression plasmids (pGp120 and pIgGp120, respectively). The gp120-Ig-coding plasmid elicited more HIV-specific $\mathrm{CD} 8^{+} \mathrm{T}$ cells and effector memory $\mathrm{CD} 8^{+}$ $\mathrm{T}$ cells than pGp120 in immunized mice. Furthermore, pGp120Ig significantly reduced the viral load after challenge with an HIV Env gp160-expressing vaccinia virus. These results represented that covalent antigen modification with an Ig sequence can modulate antigen-specific cellular immune responses [37].

On the other hand, polysaccharide and nucleic acid fraction extracted from Mycobacterium bovis bacillus Calmette-Guérin (BCGPSN) could be used as a novel adjuvant of DNA vaccine to elicit potent cellular and humoral immune responses against the HIV-1 Env antigen in $\mathrm{BALB} / \mathrm{c}$ mouse model. In this experiment, the BCG-PSN was mixed with $10 \mu \mathrm{g}$ or $100 \mu \mathrm{g}$ of DNA vaccine and injected intramuscularly two or three times. BCG-PSN co-immunization with $10 \mu \mathrm{g}$ DNA vaccine could elicit cellular and humoral immune responses which were comparable to that induced by $100 \mu \mathrm{g}$ DNA vaccine, alone. Moreover, BCG-PSN could activate TLR signaling pathways and induce Th1-type cytokines secretion. These findings suggested that BCG-PSN can be applied as a new and effective adjuvant for DNA vaccination [28].

Chemokines are largely bioactive inflammatory molecules which play a major role in a variety of immune and inflammatory responses, acting primarily as chemoattractants and activators of various leukocytes. In addition, some chemokines play a critical role in the transmission and progression of HIV-1 and 2 viruses responsible for AIDS. Recent studies have indicated that chemokines and their receptors may play an important role in the differentiation and expansion of T-cells in response to immune activation. These regulatory properties of chemokines make them suitable as molecular adjuvants [38]. For example, the modulation and regulation of immune responses was evaluated from the co-delivery of two $\beta$-chemokines as gene expression cassettes, MIP $1 \alpha$ or RANTES, along with HIV-1 DNA immunogen constructs. The data showed that MIP-1 $\alpha$ had the greatest effect on antibody responses. In addition, MIP-1 $1 \alpha$ co-expression also modulated the shift of immune responses to Th2-type (i.e., the increase of IgG1/IgG2a ratio). RANTES co-immunization also enhanced the levels of antigen-specific Th1 and CTL responses. The use of chemokine adjuvanted vaccines as HIV vaccine modulators may be important due to the interesting relationship between HIV cell entry and the receptors for $\beta$-chemokines. Indeed, $\beta$-chemokines as vaccine adjuvants increased $\beta$-chemokine production in an antigen-specific manner [38].

\section{Optimization of Motif Sequences And Promoters}

Codon-optimization of DNA from non-mammalian sources increases immunogenicity, and motif-optimization further solves the problem by optimizing short nucleotide motifs differentially found in viral and host genomes. For example, motif-optimized subtype A HIV envelope-based DNA vaccines easily elicited neutralizing antibodies, in vivo [39]. Construction of an efficient DNA vaccine depends on many variables, including composition of DNA backbone, eukaryotic gene regulatory elements, non-coding and coding regions as well as the route of delivery. Most studies on DNA-based immunization have used viral promoters to drive antigen expression $[40,41]$. The researchers have analyzed the properties of three promoters such as CMV promoter, the tissue specific murine muscle creatine kinase (MCK) promoter as well as a hybrid MCK/CMV promoter owing promoter activity and tissue specificity. The data showed that although antibody titers and cellular immune responses using the MCK construct were slightly reduced as compared to convential CMV based vector, the use of non-viral promoters may improve significantly the safety of DNA vaccines in future [40]. In addition, the studies indicated that using the muscle creatine kinase (MCK) promoter/enhancer, the efficiency of immune stimulation is strictly dependent on the ability of the HIV-1 group-specific antigen Pr55 $5^{\text {gag }}(\mathrm{Gag}$ ) released from cells. In contrast, localization of Pr55 $5^{\mathrm{gag}}$ and derivatives plays only a minor role when antigen is constitutively expressed using the viral CMV promoter [41]. In 2005, a codon-optimized hGagp17p24-Polp51 plasmid DNA expressing the HIV-1 Gag-Pol fusion protein harboring highly conserved CTL epitopes was designed. In the hGagp17p24Polp51 construct, the ribosomal frameshift site was deleted with the potentially immunosuppressive Gag nucleocapsid (p15) as well as Pol protease (p10) and integrase (p31) [42]. Immunization of HLA-A2/ $\mathrm{Kb}$ transgenic mice with the hGagp17p24-Polp51 construct induced 2- to 5-fold higher $\mathrm{CD}^{+}$T-cell responses to Gag p17-, p24-, and Pol reverse transcriptase (RT)-specific CTL epitopes than the full-length hGag-PolDFsDPr counterpart. These data were correlated with higher protection against challenge with recombinant vaccinia viruses (rVVs) expressing gag and pol gene products. Mice sera also exhibited a high titer of p24- and RT-specific IgG2 antibody responses [42].

Moreover, an AIDS vaccine was designed based on a DNA plasmid vector expressing HIV-1 recombinant gag, pol, and env genes. The production of multiple antigenic forms, including fusion-competent VLPs, by the candidate DNA vaccine constructs could provide immunological benefits for induction of protective cellular and humoral responses against HIV-1 proteins [43]. On the other hand, the effects of the conserved tyrosine-based endocytosis motif (YXXA) in the cytoplasmic domain of the HIV envelope protein (Env) were studied on its immunogenicity. Genes with codons optimized for mammalian expression were synthesized for the HIV 89.6 Env with a truncated cytoplasmic domain and a mutant Env in which the tyrosine residue in the YXXA motif was changed into a serine. Mutation of the Tyr residue enhanced surface expression of the Env protein resulting in induction of high levels of $\mathrm{T}$ cell and antibody responses. These data suggested that the $\operatorname{YXX} \varphi$ motif in the HIV Env cytoplasmic domain may play a role in virus evasion of host immune responses through affecting its immunogenicity [44]. 
A major obstacle for HIV DNA vaccine development is the genetic diversity, rapid mutation and recombination rates of HIV-1. To overcome the challenge caused by the genetic variation of HIV-1, "centralized" gene sequences were proposed to use in HIV-1 immunogen design. One of strategies generating "centralized" sequences is the use of consensus sequences. The researchers have demonstrated that the consensus HIV1 group $\mathrm{M}$ envelope genes are able to express envelope glycoproteins that retain the structure, function, and immunogenic properties of wild-type HIV-1 envelopes. Phylogenetic analysis has also shown that the consensus sequences may provide an appropriate sequence for a DNA [45]. These sequences (especially Env protein sequences) were tested as candidate vaccines in preclinical studies. To date, there have been no advanced vaccine trials in humans for evaluation and comparison of the efficacy of these centralized immunogens in various regions of the world [46].

Synthetic oligodeoxynucleotides (ODN) containing un-methylated $\mathrm{CpG}$ motifs trigger an immune response characterized by the activation of B cells, NK cells and monocytes/macrophages. It was previously shown that TLR9 participates in the recognition of CpG motifs. The motifs are internalized into acidified endosomal vesicles, and released to trigger immune stimulation through a signaling cascade involving the up-regulation of NF-kB. APCs triggered by CpG ODN up-regulate their expression of co-receptor molecules and secrete a variety of cytokines, including IL-12, IL-6, IL-1 and TNF- $\alpha$. CpG ODN has been used as vaccine adjuvants, boosting the immune response to coadministered protein and DNA based vaccines [4]. The response elicited by CpG ODN supports the induction of Th1 responses in vivo. Based on evidence that the immunogenicity of DNA vaccines can be increased using CpG motifs, 5-20 additional CpG motifs were cloned into a pUCderived plasmid. Co-administering the CpG-enriched plasmids with a DNA vaccine encoding the envelope glycoprotein of HIV (Env) to $\mathrm{BALB} / \mathrm{c}$ mice significantly increased HIV-specific cellular and humoral immunity. A significant boost was observed when the $\mathrm{CpG}$ plasmid was administered either 2 or 4 days after DNA vaccination. Plasmids containing $20 \mathrm{CpG}$ copies were the most effective immune enhancers [4].

Generally, immunization with DNA containing CpG motifs as well as synthetic CpG oligonucleotides activates the immune system through TLR9, and stimulates B cells, NK cells, and professional APCs to produce chemokines, cytokines, and immunoglobulins [47-49]. Codon optimization of DNA vaccine has been also shown to increase immunogenicity and provide better protection against Schistosoma japonicum, influenza virus, HIV, HPV, RSV, and SIV in mouse models. It should be noted that while codon optimization increases antigen expression, but this may not always enhance vaccine potency [50].

\section{Multi-plasmid DNA Vaccines}

Plasmid vaccines offer a simple strategy for combining multiple antigens in a single cocktail formulation [7]. Importantly, the combination of antigens may affect the resulting immune response. A co-vaccination strategy was used to test directly the effects of the HIV $\mathrm{Vpr}$ antigen in eliciting immune responses. The reports showed that co-vaccination with plasmids expressing HIV-1 Vpr greatly reduces antigen specific $\mathrm{CD} 8^{+}$mediated CTL activity and Th1 responses against the co-delivered antigens (Env, Gag and Pol). Indeed, a comparison of plasmid antigens as mixtures is an important step in the development of multi-antigen plasmid immunogens [7]. On the other hand, a novel animal model for testing the immunogenicity and protective immune response induced by HIV-1 DNA vaccines was developed. DBA/2 mice were immunized with GTU'-MultiHIV DNA encoding multigene for
Rev, Nef, Tat, optp17/24 and a stretch of Pol/Env epitopes. A single GTU'-MultiHIV B-clade specific plasmid or Auxo-GTU'-MultiHIVmix (mixture of four plasmids with A, B, C and FGH clade specific MultiHIV antigens) was administered via gene gun. The protective efficacy of the immune response was evaluated by challenging the mice with syngeneic tumor cells (P815) stably transfected with the MultiHIV fusion gene. The results showed that the strong MultiHIV-specific immune response generated by the GTU'-MultiHIV vaccines in DBA/2 mice was significantly able to delay the tumor growth [51]. In addition, a novel recombinant plasmid DNA therapeutic HIV-1 vaccine, GTU'multi-HIVB, containing six different genes derived from an HIV-1 subtype B isolate in untreated HIV-1 infected subjects, was safe and well tolerated with no vaccine related serious adverse events [52]. The researchers indicated that DNA vaccine can induce both specific $T$ cell responses (CTL and blast transformation) and antibodies in mice immunized with a synthetic T cell immunogen (pcDNA-TCI) derived from $\mathrm{CD} 8{ }^{+} \mathrm{CTL}$ and $\mathrm{CD} 4^{+}$Th epitopes [53].

Altogether, an important goal for a successful vaccine is the development of a vaccine with multiple HIV-1 antigens that can recognize and elicit a potent, broad, cross-reactive immune response against the virus, thus inhibiting the virus replication and immune escape. It was demonstrated that biologically attenuated Nef would be a potential immunogen in a multi-component HIV-1 vaccine [43]. Advances in determination of HIV-1 $\mathrm{CD} 8^{+} \mathrm{T}$ cell epitopes facilitate the rational design of polyepitope vaccines for eliciting broadly targeted $\mathrm{CD}^{+} \mathrm{T}$ cell responses to HIV-1. The potency of DNA vaccines consisting of ten selected HLA-A2 epitopes from the main HIV-1 antigens Env, Gag, Pol, Nef, and Vpr was evaluated in mice model. In this experiment, a number of parameters were compared including different strategies for fusing ubiquitin to the polyepitope and also spacer sequences between epitopes to optimize proteasome liberation and TAP transport. The construct with three parameters was highly immunogenic indicating the importance of some sequences for DNA vaccine development [54].

The induction of humoral and cellular immune responses to HIV-1 DNA vaccine expressing a chimeric gene of gag and gp120 (pVAX1gag-gp120) and the adjuvant effect of IFN- $\gamma$ (as a eukaryotic expression plasmid pVAX1-IFN) on HIV-1 DNA vaccine were investigated in mice model. The results indicated that co-administration of HIV-1 DNA vaccine plasmids and IFN- $\gamma$ expression plasmids can elicit stronger humoral and cellular immune responses in mice than HIV-1 DNA vaccine plasmids alone, and IFN- $\gamma$ can be an efficient immunological adjuvant in HIV DNA vaccine design [55].

\section{Heterologous Prime-Boost Strategies}

Most of the current DNA vaccines utilize CMV, $\beta$-actin, or musclespecific desmin promoters to potentiate expression of one or two fused genes of HIV-1 including the env, gag, pol, and tat. DNA vaccines comprised of multiple plasmids encoding different HIV-1 proteins have been used to obtain a broader spectrum of immunity than individual plasmids expressing single proteins. The use of these plasmid DNA vaccines proved to be safe and immunogenic in macaques, but however, these constructs needed to be boosted with viral proteins expressed by various vector systems including recombinant pox virus, modified vaccinia virus Ankara, and adenovirus for enhancing their efficiency in preventing AIDS [56].

The heterologous prime-boost regimen uses the ability of the immune system to generate large numbers of secondary antigenspecific $\mathrm{T}$ cells following an initial priming step. The same antigen is 
delivered subsequently using different vectors. Following a priming immunization, the antigen-specific $\mathrm{T}$ cell populations develop to modest levels and then reduce. Indeed, a percentage of these cells transform into antigen-specific memory $\mathrm{T}$ cells. In an heterologous boost, because the priming and boosting vectors are different, $\mathrm{T}$ cells that specifically target the viral vector are not boosted and do not activate cell number control mechanisms, therefore allowing for greater development of the disease antigen-specific $\mathrm{T}$ cell populations [11]. Several groups have now established that heterologous prime-boost regimens are the most potent strategies to induce cellular immune responses $[12,50]$. In a plasmid DNA vaccine priming and viral vector boosting regimen, the order of DNA followed by recombinant virus is important, as the reverse order did not induce higher levels of antigenspecific $\mathrm{CD}^{+} \mathrm{T}$ cells. It seems that the cytokine micro-environment created by a local virus infection during boosting is responsible for the efficient expansion of effector T cells [12]. In 2004, a consecutive immunization strategy involving priming with DNA and boosting with recombinant fowlpoxvirus ( $\mathrm{rFPV}$ ) vaccines encoding multiple common HIV-1 antigens was evaluated in 30 macaques. The vaccines were well tolerated, and a significant enhancement of DNA-vaccine primed HIV-1 specific T lymphocyte responses was observed following rFPV boosting. Co-expression of IFN $\gamma$ or IL- 12 by the rFPV vaccines did not further enhance immune responses [22]. In addition, a subtype A or B HIV gp160 plasmid DNA and Env gp140 trimeric glycoprotein coimmunization was superior to immunization with glycoprotein alone by enhancing neutralizing antibodies (NAb). These data showed that co-delivering DNA and protein can increase antibody responses to Env. Hence, this approach has the potential to simplify vaccine regimens by inducing higher antibody responses using fewer vaccinations, an advantage for a successful HIV vaccine design [57].

The reports showed that co-immunization of a DNA vaccine encoding HIV-1P24-Nef with GM-CSF in DNA priming and peptide boost strategy increases the immunogenicity of candidate vaccine. Cytokine profile studies showed that both IL- 4 and IFN- $\gamma$ levels were increased. Also, co-immunization with GM-CSF resulted in a higher level of total IgG, comprising approximately equal levels of both specific IgG1 and IgG2a subtypes. Taken together, the results suggested that GMCSF is able to induce long term memory for the HIV-1 P24-Nef vaccine candidate [58]. Recent studies have used DNA/protein or DNA/Adenovector regimens for HIV immunization. The essential mechanisms of heterogeneous prime/boost regimens are not well understood but DNA priming results in much lower antigen expression compared to protein vaccines, and this may prime T-helper cell responses with the humoral response subsequently being boosted by the high dose protein or viral vector (e.g., RV 144 tested in clinical trials) [50].

\section{The use of Apoptotic Genes}

Several studies have succeeded in enhancing the efficacy of DNA vaccines by inducing apoptosis in DNA-transfected cells [1]. For example, the reports showed that attenuated caspases induced apoptotic cell death to a certain extent and strong immune responses in a DNA flu vaccine model [1]. These studies highlighted the impact of caspase attenuation on the efficacy of apoptosis-mediated immuno-stimulation. To regulate the expression of the apoptotic gene, the bicistronic DNA vaccines were constructed that encode for HIV env and caspase-3 mutant (casp $3 \mathrm{~m}$ ) that are expressed via the encephalomyocarditis virus internal ribosomal entry site (IRES) or cytomegalovirus (CMV) promoter dependent translations. While IRES-casp $3 \mathrm{~m}$ induced weak apoptosis and caused little reduction in antigen expression, CMV-casp $3 \mathrm{~m}$ elicited strong apoptosis and led to a striking decrease in the antigen expression. Therefore, IRES-casp 3m enhanced HIV-specific immune responses, and induced significant protection against the vacciniaHIV chimeric virus. These results suggest that the appropriate level of apoptosis is important for DNA vaccine development [1]. In addition, Fas ligand is a pro-apoptotic molecule, able to stimulate death of Fas expressing cells. Fas ligand (FasL) also known as CD95L or APO-1L, is a type II membrane protein belonging to the tumor necrosis factor family of proteins and is known to induce apoptotic cell death by binding to its receptor, Fas. FasL is predominantly expressed on activated $\mathrm{T}$ and NK cells and mediates cytotoxic activity of these cells. In a study, a DNA vaccine encoding a chimeric fusion between Fas ligand and a truncated version of HIV gp120 was used as a priming vaccine, along with boosting with recombinant gp120 protein. Priming with fusion DNA resulted in a potent enhancement of immune responses to the protein boost, and, in the presence of aluminum phosphate, to a strong increase in Th2 type responses. Fas ligand delivered in a separate plasmid also had an adjuvant effect, although it was weaker than that delivered by the fusion protein [59].

\section{RNAi Technology}

RNA interference (RNAi) is a post-transcriptional gene silencing process triggered by double-stranded short hairpin RNA (shRNA) structures. RNAi has mainly been used as a research device for loss of function studies of target genes as well as a therapeutic method for human diseases. Due to its ease of production and flexibility in delivery, RNAi technology has potential applications in DNA vaccine design. One way to use RNAi for DNA vaccines would be to block genes that suppress vaccine action. For example, immune responses induced by DNA vaccines were attenuated due to the limited duration of antigen expression in vivo. Due to death of transfected cells, use of shRNA to knock down caspase 12, a cell death mediator up-regulated after DNA vaccination, resulted in increased HIV-gp120 Env antigen expression and higher $\mathrm{CD}^{+} \mathrm{T}$ cell and antibody production [50]. Similarly, RNAimediated depletion of the pro-apoptotic proteins Bak and Bax at the time of immunization of HPV16 E7 vaccine prolonged the life of antigenexpressing DCs and increased antitumor effects against E7-expressing tumors. Indeed, Fas-mediated apoptosis limited DNA vaccine-induced immune responses, and co-delivery of HPV-16 E7 DNA vaccine with DNA expressing shRNA against Fas ligand significantly enhanced CTL responses against E7. RNAi may also be used to block immunesuppressive genes inhibiting vaccine responses. Furthermore, blockade of the programmed cell death-1 (PD-1) ligand B7-H1 (PD-L1) by RNAi enhanced DC-mediated $\mathrm{T}$ cell responses and antiviral immunity in HBV transgenic mice. However, safety issues of RNAi to enhance the potency of human DNA vaccines still need to be solved and thus such technologies are likely applied to design therapeutic vaccines rather than prophylactic vaccines against various disorders [50].

\section{Clinical Trials for HIV DNA Vaccines}

Several vaccine candidates were used in different phases of clinical trials. DNA prime-viral vector boost regimens have become the primary choice for stimulation of T-cell immune responses. For example, Poxvirus vector based vaccines including Modified Vaccinia Ankara (MVA) and the genetically modified NYVAC based vaccines appeared to be efficient in inducing the immune responses and could be evaluated in combination with DNA priming in clinical trials [60]. In addition, the safety and immunogenicity of several Canarypox based vaccines with multiple HIV-1 gene inserts have been studied in humans. A phase III trial, RV144, using Canarypox (vCP1521) prime and AIDSVAX B/E boost has demonstrated modest protective 
Citation: NHabibzadeh, Bolhassani A, Vahabpour R, Sadat SM (2015) How can Improve DNA Vaccine Modalities as a Therapeutic Approach against HIV Infections? J AIDS Clin Res 6: 440. doi:10.4172/2155-6113.1000440

efficacy in Thailand. The protection in RV144 trial was short-time, and needed to use the additional boosters in participants for improving recall responses and continuing protection among them. AIDSVAX was also a component of the prime-boost (ALVAC/AIDSVAX) RV 144 vaccine in Thailand that showed successful results. In both cases, the vaccines targeted gp120 and were specific for the geographical regions. Among the Adenoviral vector vaccine candidates, replication defective Ad5 candidate indicated high immunogenicity in Phase I clinical trials and reduced viral load in the SHIV/NHP model. However, this strategy failed to prevent new infections as well as to reduce postinfection viral RNA levels in the vaccinated individuals in phase IIb. Furthermore, participants with pre-existing antibodies against Ad5 vector showed increased HIV infection rates [60]. The heterologous prime-boost strategy using DNA prime and Ad5 boost was thought to avoid the problem of pre-existing immunity. It has been shown that the pre-existing Ad5 neutralizing antibodies did not affect the levels of cell-mediated responses in the DNA/rAd5 prime-boost recipients, as compared to participants who received rAd5 alone. However, in spite of robust immune responses induced by DNA/Ad5 strategy in phase I and phase II trials, the strategy failed to show protection from new infections in a phase IIb [60]. Generally, prime-boost vaccination is an efficient approach compared to other strategies, but it still needs to develop against HIV infections in future.

\section{Conclusions}

There is considerable interest in developing plasmid DNA vaccines to prevent or treat infectious diseases, including HIV. A protective vaccine against HIV infection should elicit $\mathrm{T}$ cell immunity, protective antibody responses, and local mucosal immunity. DNA vaccines have an ability to induce humoral and cellular immune responses in animal models and humans. DNA vaccines are efficient especially as an important component of HIV vaccination strategies, typically as part of a prime/ boost vaccination strategy with viral vector or protein boost. A number of DNA prime/viral vector boost vaccines are currently being evaluated for both preclinical studies and in Phase I and Phase II clinical trials. It has been recently reported that the quality of the antigen-specific T-cell response may be more important than the magnitude of the response in the control of virus replication and disease progression. These data suggest that the induction of vaccine-specific multifunctional $\mathrm{CD} 8^{+} \mathrm{T}$ cells would be a major component of an effective vaccine. Therapeutic HIV-1 vaccines are being provided to slow disease progression and virus transmission. In addition, the generation of potent, broad neutralizing antibodies (NAbs) effective against HIV-1 from diverse modalities has remained as a key point for HIV vaccines. So far, the regimens like DNA prime/protein boost and DNA prime/viral vector boost (e.g. using adenovirus) have shown major success for eliciting potent immune responses against HIV infections.

\section{References}

1. Kojima Y, Jounai N, Takeshita F, Nakazawa M, Okuda K, et al. (2007) The degree of apoptosis as an immunostimulant for a DNA vaccine against HIV-1 infection. Vaccine 25: 438-445.

2. Bolhassani A, Yazdi SR (2009) DNA immunization as an efficient strategy for vaccination. J Med Biotechnol 1: 71-88.

3. Herrmann JE (2006) DNA vaccines against enteric infections. Vaccine 24: 3705-3708.

4. Kojima Y, Xin KQ, Ooki T, Hamajima K, Oikawa T, et al. (2002) Adjuvant effect of multi-CpG motifs on an HIV-1 DNA vaccine. Vaccine 20: 2857-2865.

5. Kuang $Y$, Zhu H, Weng X, Liu X, Chen Z, et al. (2010) Antitumor immune response induced by DNA vaccine encoding human prostate-specific membrane antigen and mouse 4-1BBL. Urology 76: 510.
6. Otten G, Schaefer M, Doe B, Liu H, Srivastava I, et al. (2004) Enhancement of DNA vaccine potency in rhesus macaques by electroporation. Vaccine 22 2489-2493.

7. Muthumani K, Kudchodkar S, Zhang D, Bagarazzi ML, Kim JJ, et al. (2002) Issues for improving multiplasmid DNA vaccines for HIV-1. Vaccine 20: 1999 2003.

8. Bolhassani A, Rafati S (2013) Mini-chaperones: potential immuno-stimulators in vaccine design. Hum Vaccin Immunother 9: 153-161.

9. Sin JI, Bagarazzi M, Pachuk C, Weiner DB (1999) DNA priming-protein boosting enhances both antigen-specific antibody and Th1-type cellular immune responses in a murine herpes simplex virus- $2 \mathrm{gD}$ vaccine model. DNA and Cell Biol 18: 771-779.

10. Marshall DJ, Rudnick KA, McCarthy SG, Mateo LR, Harris MC, et al. (2006) Interleukin-18 enhances Th1 immunity and tumor protection of a DNA vaccine. Vaccine 24: 244-253.

11. Ganguly S, Liu J, Pillai VB, Mittler RS, Amara RR (2010) Adjuvantive effects of anti-4-1BB agonist $\mathrm{Ab}$ and 4-1BBL DNA for a HIV-1 Gag DNA vaccine: different effects on cellular and humoral immunity. Vaccine 28: 1300-1309.

12. Anderson RJ, Schneider J (2007) Plasmid DNA and viral vector-based vaccines for the treatment of cancer. Vaccine 25 Suppl 2: B24-34.

13. Khan KH (2013) DNA vaccines: roles against diseases. Germs 3: 26-35

14. Pissani F, Malherbe DC, Schuman JT, Robins H, Park BS, et al. (2014) Improvement of antibody responses by HIV envelope DNA and protein coimmunization. Vaccine 32: 507-513.

15. Zhu D, Rice J, Savelyeva N, Stevenson FK (2001) DNA fusion vaccines against B-cell tumors. Trends Mol Med 7: 566-572.

16. Scott JK, Gulzar N, Klaric K, Ross TM, Lu S, et al. (2012) DNA vaccines that express the MPER of HIV-1gp41 elicit different antibodies depending upon their transmembrane and cytoplasmic domains. Retrovirology 9: P348.

17. Ivanoff LA, Dubay JW, Morris JF, Roberts SJ, Gutshall L, et al. (1992) V3 loop region of the HIV-1 gp120 envelope protein is essential for virus infectivity. Virology 187: 423-432.

18. Cristillo AD, Weiss D, Hudacik L, Restrepo S, Galmin L, et al. (2008) Persistent antibody and $T$ cell responses induced by HIV-1 DNA vaccine delivered by electroporation. Biochem Biophys Res Commun 366: 29-35.

19. Daemi A, Bolhassani A, Rafati S, Zahedifard F Hosseinzadeh S, et al. (2012) Different domains of glycoprotein 96 influence HPV16 E7 DNA vaccine potency via electroporation mediated delivery in tumor mice model. Immunol Lett 148 : 117-125.

20. Stevenson FK, Ottensmeier $\mathrm{CH}$, Rice J (2010) DNA vaccines against cance come of age. Curr Opin Immunol 22: 264-270.

21. Mölder T, Adojaan M, Kaldma K, Ustav M, Sikut R (2009) Elicitation of broad CTL response against HIV-1 by the DNA vaccine encoding artificial multicomponent fusion protein MultiHIV--study in domestic pigs. Vaccine 28: 293298.

22. Dale CJ, De Rose R, Wilson KM, Croom HA, Thomson S, et al. (2004) Evaluation in macaques of HIV-1 DNA vaccines containing primate $\mathrm{CpG}$ motifs and fowlpoxvirus vaccines co-expressing IFNgamma or IL-12. Vaccine 23: 188197.

23. Dolter KE, Evans CF, Ellefsen B, Song J, Boente-Carrera M, et al. (2011) Immunogenicity, safety, biodistribution and persistence of ADVAX, a prophylactic DNA vaccine for HIV-1, delivered by in vivo electroporation. Vaccine 29: 795-803

24. Xu F, Hong M, Ulmer JB (2003) Immunogenicity of an HIV-1 gag DNA vaccine carried by attenuated Shigella. Vaccine 21: 644-648

25. Wolchok JD, Houghton AN (2002) DNA-based cancer vaccines. Encyclopedia of Cancer 2: 73-78.

26. Wen J, Yang Y, Zhao G, Tong S, Yu H, et al. (2012) Salmonella typhi Ty21a bacterial ghost vector augments HIV-1 gp140 DNA vaccine-induced periphera and mucosal antibody responses via TLR4 pathway. Vaccine 30: 5733-5739.

27. Shinoda K, Xin KQ, Kojima Y, Saha S, Okuda K, et al. (2006) Robust HIVspecific immune responses were induced by DNA vaccine prime followed by attenuated recombinant vaccinia virus (LC16m8 strain) boost. Clin Immunol 119: 32-37. 
Citation: NHabibzadeh, Bolhassani A, Vahabpour R, Sadat SM (2015) How can Improve DNA Vaccine Modalities as a Therapeutic Approach against HIV Infections? J AIDS Clin Res 6: 440. doi:10.4172/2155-6113.1000440

28. Sun J, Hou J, Li D, Liu Y, Hu N, et al. (2013) Enhancement of HIV-1 DNA vaccine immunogenicity by BCG-PSN, a novel adjuvant. Vaccine 31: 472-479.

29. Xu R, Megati S, Roopchand V, Luckay A, Masood A, et al. (2008) Comparative ability of various plasmid-based cytokines and chemokines to adjuvant the activity of HIV plasmid DNA vaccines. Vaccine 26: 4819-4829.

30. Liu LJ, Watabe S, Yang J, Hamajima K, Ishii N, et al. (2001) Topical application of HIV DNA vaccine with cytokine-expression plasmids induces strong antigenspecific immune responses. Vaccine 20: 42-48.

31. Qiu JT, Chang TC, Lin CT, Chen YM, Li FQ, et al. (2007) Novel codon-optimized GM-CSF gene as an adjuvant to enhance the immunity of a DNA vaccine against HIV-1 Gag. Vaccine 25: 253-263.

32. Xin KQ, Hamajima K, Sasaki S, Tsuji T, Watabe S, et al. (1999) IL-15 expression plasmid enhances cell-mediated immunity induced by an HIV-1 DNA vaccine. Vaccine 17: 858-866.

33. Jiang W, Jin N, Cui S, Li Z, Zhang L, et al. (2006) Enhancing immune responses against HIV-1 DNA vaccine by coinoculating IL-6 expression vector. J Virol Methods 136: 1-7.

34. Nimal S, Heath AW, Thomas MS (2006) Enhancement of immune responses to an HIV gp120 DNA vaccine by fusion to TNF alpha cDNA. Vaccine 24: 32983308.

35. Huang Y, Chen A, Li X, Chen Z, Zhang W, et al. (2008) Enhancement of HIV DNA vaccine immunogenicity by the NKT cell ligand, alpha-galactosylceramide. Vaccine 26: 1807-1816.

36. Kanagavelu SK, Snarsky V, Termini JM, Gupta S, Barzee S, et al. (2012) Soluble multi-trimeric TNF superfamily ligand adjuvants enhance immune responses to a HIV-1 Gag DNA vaccine. Vaccine 30: 691-702.

37. Shimada M, Yoshizaki S, Jounai N, Kondo A, Ichino M, et al. (2010) DNA vaccine expressing HIV-1 gp120/immunoglobulin fusion protein enhances cellular immunity. Vaccine 28: 4920-4927.

38. Boyer JD, Kim J, Ugen K, Cohen AD, Ahn L, et al. (1999) HIV-1 DNA vaccines and chemokines. Vaccine17: S53-S64

39. Pissani F, Malherbe DC, Robins H, DeFilippis VR, Park B, et al. (2012) Motif-optimized subtype A HIV envelope-based DNA vaccines rapidly elicit neutralizing antibodies when delivered sequentially. Vaccine 30: 5519-5526.

40. Bojak A, Hammer D, Wolf $\mathrm{H}$, Wagner R (2002) Muscle specific versus ubiquitous expression of Gag based HIV-1 DNA vaccines: a comparative analysis. Vaccine 20: $1975-1979$

41. Bojak A, Wild J, Wolf $H$, Wagner $R$ (2002) Efficiency of a myogenic DNA vaccine is strictly dependent upon cellular localization of HIV-1 Pr55(gag). Vaccine 20: 1980-1984.

42. Bolesta E, Gzyl J, Wierzbicki A, Kmieciak D, Kowalczyk A, et al. (2005) Clustered epitopes within the Gag-Pol fusion protein DNA vaccine enhance immune responses and protection against challenge with recombinant vaccinia viruses expressing HIV-1 Gag and Pol antigens. Virology 332: 467-469.

43. Majumder B, Gray B, McBurney S, Schaefer TM, Dentchev T, et al. (2003) Attenuated nef DNA vaccine construct induces cellular immune response: role in HIV-1 multiprotein vaccine. Immunology Letters 89: 207-214.

44. Bu Z, Ye L, Vzorov A, Taylor D, Compans RW, et al. (2004) Enhancement of immunogenicity of an HIV Env DNA vaccine by mutation of the Tyr-based endocytosis motif in the cytoplasmic domain. Virology 328: 62-73.

45. Yan J, Corbitt N, Pankhong P, Shin T, Khan A, et al. (2011) Immunogenicity of a novel engineered HIV-1 clade C synthetic consensus-based envelope DNA vaccine. Vaccine 29: 7173-7181.
46. Tongo M, Burgers WA (2014) Challenges in the design of a T cell vaccine in the context of HIV-1 diversity. Viruses 6: 3968-3990.

47. Coban C, Ishii KJ, Gursel M, Klinman DM, Kumar N (2005) Effect of plasmid backbone modification by different human $\mathrm{CpG}$ motifs on the immunogenicity of DNA vaccine vectors. J Leukoc Biol 78: 647-655.

48. Coban C, Kobiyama K, Aoshi T, Takeshita F, Horii T, et al. (2011) Novel strategies to improve DNA vaccine immunogenicity. Curr Gene Ther 11: 479-484.

49. Yu YZ, Li N, Ma Y, Wang S, Yu WY, et al. (2013) Three types of human CpG motifs differentially modulate and augment immunogenicity of nonviral and viral replicon DNA vaccines as built-in adjuvants. Eur J Immunol 43: 228-239.

50. Li L, Saade F, Petrovsky N (2012) The future of human DNA vaccines. J Biotechnol 162: 171-182

51. Malm M, Sikut R, Krohn K, Blazevic V (2007) GTU-MultiHIV DNA vaccine results in protection in a novel P815 tumor challenge model. Vaccine 25: 32933301.

52. Vardas E, Stanescu I, Leinonen M, Ellefsen K, Pantaleo G, et al. (2012) Indicators of therapeutic effect in FIT-06, a Phase II trial of a DNA vaccine GTU(囚)-Multi-HIVB, in untreated HIV-1 infected subjects. Vaccine 30: 40464054 .

53. Bazhan SI, Belavin PA, Seregin SV, Danilyuk NK, Babkina IN, et al. (2004) Designing and engineering of DNA-vaccine construction encoding multiple CTL-epitopes of major HIV-1 antigens. Vaccine 22: 1672-1682.

54. Bazhan SI, Karpenko LI, Ilyicheva TN, Belavin PA, Seregin SV, et al. (2010) Rational design based synthetic polyepitope DNA vaccine for eliciting HIVspecific CD8+ T cell responses. Mol Immunol 47: 1507-1515.

55. Jiang W, Ren L, Jin N (2007) HIV-1 DNA vaccine efficacy is enhanced by coadministration with plasmid encoding IFN-alpha. J Virol Methods 146: 266 273.

56. Liu Z, Singh DK, Sheffer D, Smith MS, Dhillon S, et al. (2006) Immunoprophylaxis against AIDS in macaques with a lentiviral DNA vaccine. Virology 351: 444-454.

57. Pissani F, Malherbe DC, Schuman JT, Robins H, Park BS, et al. (2014) Improvement of antibody responses by HIV envelope DNA and protein coimmunization. Vaccine 32: 507-513

58. Mahdavi M, Ebtekar M, Khorram Khorshid HR, Azadmanesh K, Hartoonian C et al. (2011) ELISPOT analysis of a new CTL based DNA vaccine for HIV-1 using GM-CSF in DNA prime/peptide boost strategy: GM-CSF induced longlived memory responses. Immunol Lett 140: 14-20.

59. Huang B, Mao CP, Peng S, Hung CF, Wu TC (2008) RNA interference-mediated in vivo silencing of fas ligand as a strategy for the enhancement of DNA vaccine potency. Hum Gene Ther 19: 763-773.

60. Shete A, Thakar M, Mehendale S, Paranjape R (2014) Is prime boost strategy a promising approach in HIV vaccine development? J AIDS Clin Res 5:293. 